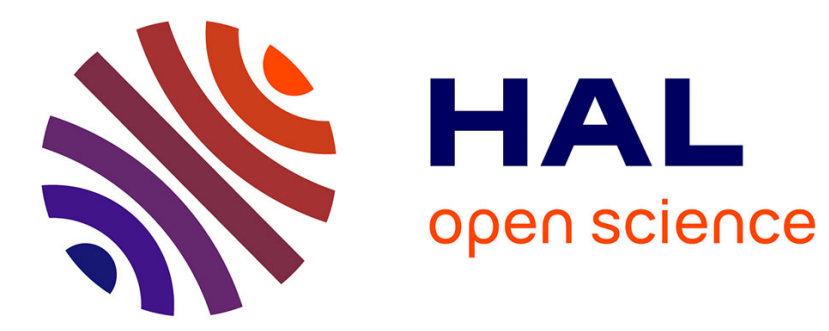

\title{
Instabilities under mechanical tension in a smectic cylinder
}

\author{
P.-G. de Gennes, P. Pincus
}

\section{To cite this version:}

P.-G. de Gennes, P. Pincus. Instabilities under mechanical tension in a smectic cylinder. Journal de Physique, 1976, 37 (11), pp.1359-1361. 10.1051/jphys:0197600370110135900 . jpa-00208533

\section{HAL Id: jpa-00208533 https://hal.science/jpa-00208533}

Submitted on 1 Jan 1976

HAL is a multi-disciplinary open access archive for the deposit and dissemination of scientific research documents, whether they are published or not. The documents may come from teaching and research institutions in France or abroad, or from public or private research centers.
L'archive ouverte pluridisciplinaire HAL, est destinée au dépôt et à la diffusion de documents scientifiques de niveau recherche, publiés ou non, émanant des établissements d'enseignement et de recherche français ou étrangers, des laboratoires publics ou privés. 


\title{
INSTABILITIES UNDER MECHANICAL TENSION IN A SMECTIC CYLINDER
}

\author{
P. G. DE GENNES and P. PINCUS (*) \\ Collège de France, 75231 Paris Cedex 05, France
}

(Reçu le 1er juin 1976, accepté le 23 juin 1976)

\begin{abstract}
Résumé. - On calcule le seuil d'instabilité de Helfrich-Hurault pour un smectique en géométrie cylindrique (couches disposées en cylindres de révolution coaxiaux). Lorsque les couches sont mises sous tension, par exemple par un pulse thermique de signe convenable, il apparait une instabilité d'ondulation avec un vecteur d'onde optimal $q^{*}$. Mais la variation radiale de la distorsion est bien plus complexe que dans le cas habituel d'un spécimen plan. Il se peut que $q^{*}$ soit révélé par la texture en perles observée sur CBOOA par Cladis et White.

Abstract. - We discuss theoretically the analog of the Helfrich-Hurault instability for a smectic cylinder (as opposed to the usual case of a flat slab). The smectic layers are initially in a ideal concentric cylinder conformation. It is then assumed that (using for instance a temperature pulse of adequate sign) the layers are put under tension. Then an undulation instability can set in, at a certain optimal wave vector $q^{*}$ parallel to the cylinder axis. We discuss the distortion field at threshold : it is significantly more complex than in the slab problem. The wave vector $q^{*}$ might be revealed in the beaded textures observed by Cladis and White in smectic cylinders of CBOOA.
\end{abstract}

1. Introduction. - Recent observations [1] of smectic liquid crystals in the form of macroscopic cylinders with homeotropic boundaries have demonstrated the existence of a line of beads (periodic defects) along the axis. These results have been interpreted [2] in terms of thermodynamic fluctuations of the smectic layer displacement field $u(\mathbf{r})$ leading to the prediction that the bead radius $r_{0}$ should vary as

$$
r_{0} \cong \pi(2 R \lambda)^{1 / 2} \text {. }
$$

Here $R$ is radius of the cylinder and $\lambda=\left(K_{1} / B\right)^{1 / 2}$; $K_{1}$ is the splay elastic constant and $B$ the elastic modulus associated with compression of the smectic layers [3]. The parameter $\lambda$ has dimensions of length and is typically of the order of molecular dimensions, i.e. $\lambda \sim 20 \AA$.

Eq. (1) apparently gives the right order of magnitude for the bead periodicity. However, the picture is based on a very unusual notion : namely that the thermal fluctuations of a cylindrical texture may be spontaneously large enough to generate permanent defects - the latter being then frozen in by some unexplained means.

Our approach is slightly different : we assume that at some moment during the sample preparation, the smectic layers are put under tension. For instance,

(*) Alfred Guggenheim Fellow supported in part by the C.N.R.S the N.S.F., and O.N.R.

Permanent address : Department of Physics, Univ. of California, Los Angeles. in cases where the layer thickness $d(T)$ increases more rapidly with the temperature $T$, than the radius $R$ of the container, a rapid cooling will provide the tension. (If, on the other hand, $d(T)$ decreases with $T$ we would require a positive temperature jump.)

In any case, if the $T$ jump is fast enough, we may have an undulation instability, reminiscent of what is known for smectic slabs [3]. If the amplitude of the oscillation is large enough, the distorted structure may switch to permanent defects : our assumption is that the repeat period of the defects coincides with the repeat period of the original smooth undulation : We study the onset by standard elasticity theory. The opposite regime of developed instability and the precise nature of the defects (focal conics or more special deformations) have been discussed by Kleman [4].

The analysis of onset is, however, much more delicate than for the slab problem. Two features will be seen to be essential :

a) The deformed sample just below threshold is not uniformly dilated but the dilation decreases with the distance $r$ from the axis like $1 / r$.

b) The unstable mode is not uniform in space, and is somewhat concentrated in the central region.

2. Deformations. - Let $r, \varphi$, and $z$ be semi-polar axes for the cylinder. We consider a layer displacement $u(r, \varphi, z)$ which, for the case of interest, will depend only on $r$ and $z$. 
The elastic free energy may be put under the form

$$
F / B=\frac{1}{2}\left(\frac{\partial u}{\partial r}\right)^{2}+\frac{1}{2} \lambda^{2}\left(\nabla^{2} u-\frac{1}{r}\right)^{2}
$$

where $u(\mathbf{r})$ is the layer deformation which balances the splay elastic energy created by the constraint of the cylindrical geometry. The free energy is minimized if the displacement satisfies

$$
-\nabla_{r}^{2} u+\lambda^{2} \nabla^{2}\left(\nabla^{2} u-\frac{1}{r}\right)=0
$$

where

$$
\nabla_{r}^{2}=\frac{1}{r} \frac{\mathrm{d}}{\mathrm{d} r}\left(r \frac{\mathrm{d}}{\mathrm{d} r}\right)
$$

2.1 THE SOLUTION BeLOW THRESHOLD. - Let us first consider solutions of the form $u=u_{0}(r)$; i.e. a purely radial displacement field with no axial undulations. This will describe the deformed state prior to any longitudinal instability. For $r \gg \lambda$, it is easy to verify that $\nabla^{2} u_{0}-1 / r \rightarrow 0$. This in turn leads to

$$
u_{0}(r) \cong c \ln r-\lambda^{2} / r
$$

where $c$ is a constant which determines the overall strain of the system. (It will ultimately be shown that as soon as $c$ exceeds a critical value $c^{*} \sim \lambda$ the axial instability will set in.) The dominant term in (4) for all physical $r(r \gg \lambda)$ is the $\log$ term. The tension at the outer surface is

$$
\tau=\left.B \frac{\partial u}{\partial r}\right|_{r=R}=\frac{B c}{R} .
$$

For a given $\tau$ this fixes the constant $c$.

2.2 SMall UNDUlation THEORY. - Let us now put $u(r, z)=u_{0}(r)+w(r, z)$ where (near threshold) $w(r, z)$ is assumed to be small compared to $u_{0}(r)$. Note that we consider only the modes without any $\varphi$ dependence. The free energy density analogous to eq. (2) is then $F=F_{\text {comp }}+F_{\text {splay }}$ where the compressional contribution is

$$
\begin{aligned}
F_{\text {comp }} / B & =\frac{1}{2}\left(\frac{\partial u_{0}}{\partial r}+\frac{\partial w}{\partial r}\right)^{2}-\frac{1}{2}\left(\frac{\partial u_{0}}{\partial r}\right)\left(\frac{\partial w}{\partial z}\right)^{2} \\
& \cong \frac{1}{2}\left(c / r+\frac{\partial w}{\partial r}\right)^{2}-\frac{1}{2} c / r\left(\frac{\partial w}{\partial z}\right)^{2}
\end{aligned}
$$

The second non-linear term corresponds to the dilation of the smectic plane thickness as the planes are tilted [3]. The splay free energy density is

$$
\begin{aligned}
F_{\text {splay }} / B & =\frac{1}{2} \lambda^{2}\left(\nabla^{2} u_{0}-\frac{1}{r}+\nabla^{2} w\right)^{2} \\
& \cong \frac{1}{2} \lambda^{2}\left(\nabla^{2} w-\frac{1}{r}\right)^{2} .
\end{aligned}
$$

Using the fact that $u_{0}$ satisfies eq. (3), the net contribution to the free energy density arising from the perturbation $w$ is

$$
\Delta F / B=\frac{1}{2}\left(\frac{\partial w}{\partial r}\right)^{2}-\frac{1}{2}(c / r)\left(\frac{\partial w}{\partial z}\right)^{2}+\frac{1}{2} \lambda^{2}\left(\nabla^{2} w\right)^{2}
$$

or assuming a sinusoidal axial variation

$$
\begin{aligned}
w & =\mathrm{e}^{i q z} \rho(r), \\
2 \Delta F / B & =[\nabla \rho(r)]^{2}-(c / r) q^{2} \rho^{2}(r)+ \\
& +\lambda^{2}\left(\nabla_{r}^{2} \rho-q^{2} \rho\right)^{2} .
\end{aligned}
$$

The incremental contribution to the free energy is an extremum when

$-\nabla_{r}^{2} \rho-\left(c q^{2} / r\right) \rho+\lambda^{2}\left(\nabla_{r}^{2}-q^{2}\right)^{2} \rho=0$.

This is essentially an eigenvalue problem $\left(\lambda^{2} q^{4}\right.$ is the eigenvalue) whose solution for homeotropic boundary conditions $[\rho(R)=0]$ leads to a relationship between $c$, the strain for the purely radial solution, and the sinusoidal wave vector $q$. If the function $c(q)$ has a minimum $c^{*}$ for some value of the wave vector $q^{*}$, this then defines the period of first instability.

2.3 WKB APPROXIMATION. - In order to first obtain a rough idea of the lowest energy (minimum number of nodes in $\rho(r)$ ) solution to (11), let us first use a WKB type method. If we suppose that the function $\rho(r)$ is localized within a length $\xi$, then eq. (11) becomes

$+\xi^{-2}\left(1+2 \lambda^{2} q^{2}\right)-\left(c q^{2} / \xi\right)+\lambda^{2} q^{4} \cong 0$

where we have neglected the small $\nabla_{r}^{4}$ term (this approximation may be verified a posteriori). Solving for $c(q, \xi)$,

$$
c \cong(\lambda q)^{2} \xi+2 \lambda^{2} / \xi+\left(\xi q^{2}\right)^{-1}
$$

which is minimum with respect to $q^{2}$ for $q^{*}=(\lambda \xi)^{-1 / 2}$ with $c^{*}=2 \lambda(1+\lambda / \xi)$. Thus, $c^{*}$ is an absolute minimum for the largest possible localization length $\xi$. But $\xi_{\max } \sim R$, leading to $q^{*} \sim(\lambda R)^{-1 / 2}$ and $c^{*} \sim 2 \lambda$. This result is in essential agreement with the observations $[1,2]$ on the radius dependence of the bead dimensions. (Note that the neglected $\nabla_{r}^{4}$ term gives an additional contribution to $c$ (eq. 13) of order $\lambda^{2} \xi^{-3} q^{-2} \sim \lambda(\lambda / \xi)^{2}$ which is smaller by $(\lambda / R)$ than the smallest term retained.

2.4 COMPLETE FORM OF THE UNSTABle MODE. We now present a more rigorous solution to eq. (11), still neglecting the $\nabla_{r}^{4}$ contribution. Changing variables by,

$$
\begin{aligned}
\rho(r) & =\mathrm{e}^{-z / 2} F(z) \\
z & =2 \lambda q^{2}\left(1+2 \lambda^{2} q^{2}\right)^{-1 / 2} r \\
a & =\frac{1}{2}\left[1-(c / \lambda)\left(1+2 \lambda^{2} q^{2}\right)^{-1 / 2}\right] .
\end{aligned}
$$


Eq. (11) becomes

$$
z \frac{\mathrm{d}^{2} \mathrm{~F}}{\mathrm{~d} z^{2}}+(1-z) \frac{\mathrm{d} F}{\mathrm{~d} z}-a F=0,
$$

which is the confluent hypergeometric eq. [5]. The solution regular at the origin is $F(z)=F(a|1| z)$, the confluent hypergeometric function. The boundary condition $\rho(R)=0$, leads to a relationship $c(q)$. For $a<0, F(a|1| z)$ has zeros which occur at $z \gg 1$ if $|a| \gg 1$. Then using the asymptotic expression for $F(a|1| z)$, [5] we have

$$
F(a|1| b) \cong 1+a \mathrm{e}^{b} / b+\cdots=0 .
$$

We may write [using eq. (14)]

$$
c / \lambda \cong(1-2 a)[1+(b \lambda / 2 R)]
$$

[for $b \lambda / R \ll 1$ ], where $b$ and $a$ are related by the eigenvalue eq. (16) to give

$$
a \simeq b \mathrm{e}^{-b} .
$$

Minimizing $c(b)$ and using eq. (14) leads to

$$
\begin{aligned}
\left(q^{*}\right)^{2} & \cong(\ln 4 R / \lambda)(2 \lambda R)^{-1}, \\
c^{*} & \simeq \lambda\left[1-\frac{3}{2}(\lambda / R) \ln 4(R / \lambda)\right] .
\end{aligned}
$$

This, apart from the logarithmic correction is in substantial accord with the WKB estimate. The aspect of the solution at threshold is shown on figure 2 .

3. Conclusions. - 1) The calculation presented above demonstrates the existence of an undulation instability under tension. In ref. [2] no such instability was found. The essential difference between the two investigations is that ref. [2] neglected the small, but crucial, $(\lambda q)^{2} \nabla_{r}^{2} \rho$ contribution to eq. (11).

2) We conjecture that the bead texture is an image of this unstability, corresponding to situations far above threshold. The period $2 \pi / q^{*}$ suggested by eq. (17) is not very different from the simple Cladis estimate and appears thus compatible with the data. There remains to show that the sign of $\frac{d}{d T} d(T)$ and the thermal cycling history are compatible with our model.

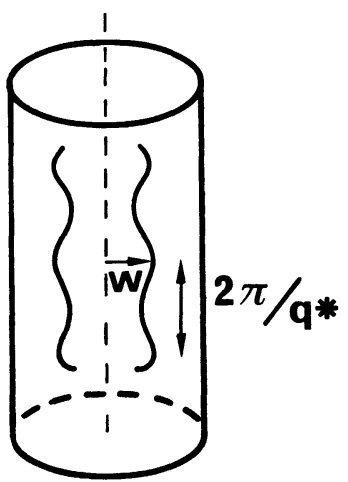

Fig. 1. - A sketch of the layer displacement field $w(r)$ near threshold. The field is axially symmetric.

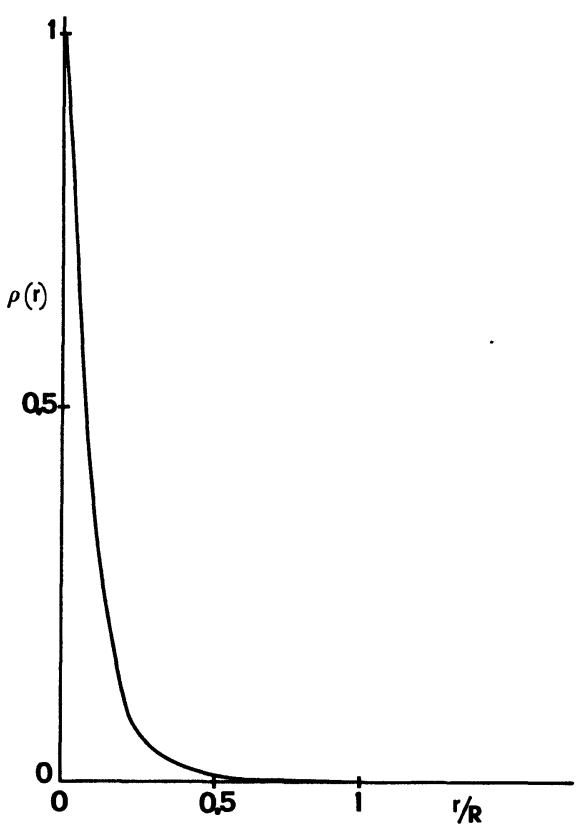

FIG. 2. - The radial dependence $\rho(r)$ of the displacement field for $\ln 4 R / \lambda \simeq 20$.

3) More systematic experiments could be performed driving the instability by a mechanical means rather than by thermal pulses - imposing for instance a pressure difference between the inside and the outside of the container.

Acknowledgments. - We would like to acknowledge helpful discussions with $\mathbf{M}$. Veyssié and M. Kléman. S. Pincus has kindly helped with some of the calculational aspects.

\section{References}

[1] Cladis, P. E., Phil. Mag. 29 (1974) 641.

Cladis, P. E. and White, A. E., to be published in J.A.P.

[2] Cladis, P. E., J. Physique Colloq. 37 (1976) C3-137.

[3] See De Gennes P. G., The Physics of Liquid Crystals (Clarendon Press, Oxford), 1974, chapter 7.
[4] Kléman, M., Phil. Mag. to be published.

[5] JAHNKE-EMDE-LösCH, Tables of High Functions (McGraw-Hill, New York), 1960, chapter XI. 